\title{
Resultados terapéuticos en pacientes acromegálicos: es tiempo de intervenir
}

\author{
Carmen Carrasco $\mathbf{M}^{1}$, Jesús Véliz $L^{1}$, D avid Rojas $Z^{2}$, \\ Nelson W ohllk G ${ }^{1}$.
}

\section{Results of surgical treatment for acromegaly in 53 patients}

Background: The treatment of choice for acromegaly is surgery that, according to the literature, is curative in $91 \%$ of pituitary microadenomas and $73 \%$ of macroadenomas. Aim: To report the results of surgical treatment in 53 patients with acromegaly. Material and methods: Retrospective review of medical records of all patients with acromegaly, operated between 1984 and 2004. When necessary, patients were contacted by telephone to complete information or to perform biochemical or imaging studies. A normal value of insulin like growth factor I (IGF-1) for age and sex, a growth hormone (GH) nadir of less than $1 \mathrm{ng} / \mathrm{ml}$ during a glucose tolerance test or a basal GH of less than $2.5 \mathrm{ng} / \mathrm{ml}$, all assessed three months after surgery, were considered as criteria for cure. Results: Biochemical cure was achieved in $67 \%$ of patients with pituitary microadenomas and $21 \%$ of patients with macroadenomas. In $47 \%$ of patients with neuro-ophtalmological involvement, a partial or total recovery in the visual field defect was achieved. The most common surgical complications were transient diabetes insipidus in 19\%, persistent diabetes insipidus in $4 \%$ and cerebrospinal fluid fistula in $4 \%$. A lower size of the tumor and lower preoperative growth hormone levels were associated with a better chance of cure. Conclusions: The cure rates obtained in this group of patients are clearly lower than those reported abroad. These results stress the importance of having a national registry of acromegaly and the need to train neurosurgeons in the treatment of pituitary tumors (Rev Méd Chile 2006; 134: 989-96).

(Key words: Acromegaly; Neurosurgery; Pituitary neoplasms)

Recibido el 22 de agosto, 2005. Aceptado el 20 de enero, 2006.

${ }^{1}$ Sección Endocrinología, Hospital del Salvador. Facultad de Medicina, Universidad de Chile, División Oriente. ${ }^{2}$ Instituto de Neurocirugía Asenjo, Facultad de Medicina Universidad de Chile, División Oriente. Santiago de Chile.

$\mathrm{L}^{2}$ a acromegalia es una enfermedad poco común, que tiene una incidencia anual estimada de 3 a 4 casos por millón de habitantes ${ }^{1,2}$. En $95 \%$ de los

Correspondencia a: Carmen Carrasco M. Departamento de Endocrinología, Pontificia Universidad Católica de Chile. Lira 85, 5ํㅜ piso. E mail: cantonie@med.puc.cl casos es causada por un adenoma hipofisiario productor de hormona del crecimiento (GH), el cual produce síntomas relacionados tanto con los efectos metabólicos de GH y su mediador IGF-I, como con el efecto local de la masa tumoral. El diagnóstico de acromegalia implica para el afectado una mayor morbilidad, una tasa de mortalidad 2 a 4 
veces superior a la esperada, con una reducción en su pronóstico de sobrevida en aproximadamente 10 años $^{3,4}$. Los estudios epidemiológicos han demostrado que es posible recuperar índices de mortalidad similares a la población general, si se logra reducir las concentraciones de GH bajo 2,5 $\mathrm{ng} / \mathrm{ml}$ o disminuir los niveles de IGF-I a valores adecuados para edad y sexo, sin importar el tiempo de exposición previa a la enfermedad ${ }^{3,5}$. De esto se desprende la importancia de contar con médicos alertas a la sospecha clínica, acceso a endocrinólogos que confirmen el diagnóstico y neurocirujanos expertos que puedan resolver adecuadamente esta patología.

El tratamiento de elección sigue siendo la cirugía, con tasas de control de la enfermedad en centros de referencia extranjeros que llegan a 91\% para microadenomas y $73 \%$ para macroadenomas $^{6,7}$. Estas cifras son aún insuficientes, motivo por el cual los esfuerzos se concentran en perfeccionar terapias complementarias, como el uso de análogos de somatostatina, antagonistas del receptor de GH o nuevas técnicas de radioterapia.

A nivel nacional, los resultados quirúrgicos parecieran ser inferiores a los internacionales. Las publicaciones, en a lo menos 2 centros universitarios, muestran características clínicas y tasas de curación variables, pero cuentan con un número reducido de pacientes evaluados o utilizan criterios antiguos de curación ${ }^{8,9}$. El Instituto de Neurocirugía Asenjo (INCA) cuenta con un flujo de 140 tumores hipofisiarios operados anualmente. Nuestro propósito fue conocer la eficacia del tratamiento quirúrgico de la acromegalia en el INCA en el período 1984-2004, mediante una revisión de fichas, aplicando un protocolo de revisión estandarizado a la luz de los nuevos criterios y conocer el efecto de variables de exposición sobre el resultado terapéutico.

\section{PACIENTES Y MÉTODO}

Pacientes. Se realizó un estudio retrospectivo aplicando un protocolo de revisión estandarizado, basado en la publicación de Drange et $\mathrm{al}^{10}$. Se incluyeron pacientes acromegálicos operados en el INCA entre los años 1984 y 2004, los cuales fueron reclutados en base a los registros de sus médicos tratantes, revisión de tablas quirúrgicas y pacientes en control actual en el policlínico de neuroendocrinología. En caso necesario, se contactó telefónicamente a los pacientes para completar la información o realizar exámenes bioquímicos y de imágenes. Se analizaron 53 pacientes, los cuales tenían evidencias clínicas y bioquímicas de acromegalia, así como una masa tumoral selar en el estudio radiológico, a excepción de 3 casos en los cuales el tumor sólo se encontró durante la cirugía.

El registro incluyó variables como edad, sexo, tiempo de evolución de la sintomatología previo al diagnóstico, especialista que sospechó el diagnóstico y síntomas. Se registró estudio hormonal, neuroftalmológico y radiológico centralizados pre y posoperatorios. El seguimiento posoperatorio incluyó una evaluación a los 3 meses y luego anualmente, a excepción de 2 casos en los cuales no fue posible contar con esta evaluación. En los casos en los que se indicó radioterapia, ésta fue convencional y realizada en diferentes centros, por lo cual no contamos con las especificaciones técnicas. No se encontró un criterio común para su prescripción, la cual se basó sólo en la decisión del tratante.

El tamaño tumoral se determinó en base al estudio radiológico con tomografía axial computarizada (TAC) o Resonancia Nuclear Magnética (RNM) selar. Se consideró el diámetro mayor para efectos de clasificarlos en micro o macroadenomas. La sintomatología evaluada se eligió en base a lo publicado en la literatura como prevalente. Se intermogó dirigidamente sobre antecedentes familiares de tumores pituitanios, paratimideos o pancreáticos y se midió calcemia en todos los pacientes. La sintomatología de hipogonadismo incluyó disminución de la líbido, oligo o amenormea, infertilidad o disfunción eréctil. El diagnóstico de hipopituitarismo incluyó hipogonadismo hipogonadotrópico (testosterona total o estradiol bajo con LH o FSH inapropiadamente normal o baja), hipotiroidismo secundario (T4 libre baja con TSH inapropiadamente normal o baja) o hipocortisolismo secundario (cortisol basal $<3 \mu \mathrm{g} / \mathrm{dl}$ o estimulado con Synacthen $\left.{ }^{\circledR}<15 \mu \mathrm{g} / \mathrm{dl}\right)^{11}$. Se consideró hiperprolactinemia valores de prolactina superiores a $40 \mathrm{ng} / \mathrm{ml}^{12}$.

Se definió como curado aquel paciente que a los tres meses después de la cirugía presentó una IGF-I normal para edad y sexo o frenaba $\mathrm{GH}<1$ $\mathrm{ng} / \mathrm{ml}$ en el test de tolerancia a la glucosa oral $(75 \mathrm{~g}$ glucosa, TTGO) o en su defecto, contaba con GH basal $<2,5 \mathrm{ng} / \mathrm{ml}$, tomada aleatoriamente. Esto 
último fue adoptado ya que algunos de los pacientes más antiguos sólo contaban con GH basal y este valor se correlacionaría con normalización de la mortalidad $^{3,13}$. En 43 pacientes contamos con $\mathrm{GH}$ basal a las $48 \mathrm{~h}$ poscirugía y en 51 pacientes con el seguimiento posoperatorio.

Método. El estudio hormonal fue realizado en el laboratorio IEMA y consistió en la medición sérica de T4 libre/TSH, cortisol basal, prolactina, LH/ testosterona o FSH/ estradiol, IGF-I, GH basal y con TTGO. Debido a que la medición de IGF-I se inició en 1997, sólo 41\% de los pacientes cuentan con esta determinación.

La medición de GH fue realizada por dos ensayos diferentes: antes de 2001, por radioinmunoanálisis (RIA, Diagnostic Products Corporation, DPC $^{\circledR}$ ) de doble anticuerpo, siendo la sensibilidad analítica del método de 0,9 $\mathrm{ng} / \mathrm{ml}$. El coeficiente de variación (CV) intra e interensayo para un valor de $6,0 \mathrm{ng} / \mathrm{ml}$ fue de $1,5 \%$ y $1,8 \%$, respectivamente. Desde 2001 se efectuó por quimioluminiscencia (Immulite ${ }^{\circledR}$ ), siendo la sensibilidad analítica de detección de 0,01 ng/ml. El CV intra e interensayo para un valor de $7,8 \mathrm{ng} / \mathrm{ml}$ fue de $6,0 \%$ y $5,5 \%$, respectivamente. Dado que no existe un factor de conversión claro entre ambas técnicas especificada por el proveedor, se decidió adoptar lo realizado por otros autores y asumir que los valores por quimioluminiscencia son $10 \%$ superiores a los del RIA ${ }^{14,15}$.

La medición de IGF-I se realizó por inmunoradiometría (IRMA, Inmunotech ${ }^{\circledR}$ ) con límite de detección de $12 \mathrm{ng} / \mathrm{ml}$. El CV intra e interensayo para un valor de $361 \mathrm{ng} / \mathrm{ml}$ fue de 5,7\% y 8,6\% y para un valor de $1.211 \mathrm{ng} / \mathrm{ml}$ fue de $7,1 \%$ y $16,7 \%$, respectivamente.

Estadística. El análisis estadístico incluyó prueba t-de student para dos medias, pruebas no paramétricas para muestras independientes, test $\chi^{2}$ para variables categóricas (significativo $p$ $<0,005)$; utilizando el software estadístico para Windows SPSS 11.5.

\section{Resultados}

De los 53 pacientes registrados, 66\% eran mujeres y $34 \%$ hombres. La presentación como macroadenoma fue más frecuente, tanto en hombres
(88,9\%) como en mujeres (77,1\%). En la Tabla 1 se enumera la clínica más prevalente, destacando el crecimiento acral y maxilofacial, la cefalea y la hiperhidrosis. El promedio de edad de presentación fue de $41,9 \pm 13,8$ años, con un mayor número en la $4^{\text {a }}$ década. La latencia entre el inicio de la sintomatología y el diagnóstico fue de 68,9 meses (rango: 6-360), y éste fue sospechado en $24,5 \%$ de los casos por médico general, $15,1 \%$ por neurólogo, 13,2\% por endocrinólogo y 7,5\% por ginecólogo.

Del grupo total destacaban la alta frecuencia de macroadenomas (81,1\%), 32\% de hipopituitarismo y $28,3 \%$ de hiperprolactinemia. En 17/53 pacientes se encontró compromiso neuroftalmológico de grado variable, con dos casos de diplopia. Dos pacientes debutaron con apoplejía pituitaria. La Tabla 2 resume las características preoperatorias de los pacientes según tamaño tumoral. Los valores de $\mathrm{GH}$ basal fueron significativamente más altos para los macroadenomas ( $p<0,002$ ); mientras que las concentraciones de IGF-I fueron similares en ambos grupos.

Llamó la atención una paciente con IGF-I repetidamente normal, a pesar de la clínica compatible con acromegalia, presencia de un macroadenoma en la RNM y falta de frenación de GH. Se descartó insuficiencia hepática y uso de estrógenos, y los valores de IGF-I se elevaron durante el seguimiento. Pudimos diagnosticar una paciente con neoplasia endocrina múltiple tipo 1 y una acromegalia familiar aislada.

El 94,6\% de las cirugías fueron vía transesfenoidal, 1 por vía transcraneana y 2 transpterionales, logrando la curación bioquímica mediante cirugía en $28,3 \%$ de los pacientes. Estas cirugías fueron efectuadas por un total de 19 neurociruja-

\section{Tabla 1. Síntomas y signos presentes al momento del diagnóstico}

\begin{tabular}{|lc|}
\hline Clínica & Porcentaje (\%) \\
\hline Crecimiento acral o maxilofacial & 98,1 \\
Cefalea & 47,2 \\
Sudoración & 34,0 \\
Hipogonadismo & 28,3 \\
Poliartralgias & 28,3 \\
Galactorrea & 7,5 \\
\hline
\end{tabular}


Tabla 2. C aracterísticas preoperatorias de 53 acromegálicos según tamaño tumoral

\begin{tabular}{|lccc|}
\hline Parámetro & $\begin{array}{c}\text { Macroadenomas } \\
(43)\end{array}$ & $\begin{array}{c}\text { Microadenomas } \\
(10)\end{array}$ & $\mathrm{p}$ \\
\hline Sexo M/F & $1 / 1,7$ & $1 / 4$ & $\mathrm{NS}$ \\
Edad (años) (promedio $\pm \mathrm{DE})$ & $39,6 \pm 13,2$ & $51,9 \pm 12,3$ & $\mathrm{NS}$ \\
Duración (meses) & $66,16 \pm 59,4$ & $79,2 \pm 33,1$ & $\mathrm{NS}$ \\
Promedio GH \pm DE & $32 \pm 20,5$ & $18,8 \pm 6,1$ & $<0,002$ \\
Promedio IGFI \pm DE (ng/ml) & $987 \pm 451$ & $957 \pm 310$ & NS \\
PRL >40 ng/ml n (\%) & $32,6 \%$ & $10 \%$ & NS* \\
Hipopituitarismo & $37,2 \%$ & $10 \%$ & NS* \\
Compromiso NOF & $32,6 \%$ & $10 \%$ & NS* \\
\hline
\end{tabular}

NS: no significativo

Valores normales: $\mathrm{GH}>5 \mathrm{ng} / \mathrm{ml}$; prolactina $>25 \mathrm{ng} / \mathrm{ml}$, los valores normales de IGF-1 son ajustados por sexo y edad según cada paciente.

*La ausencia de diferencias estadísticamente significativas podría explicarse por el reducido número de microadenomas.

nos diferentes. Tal como se observa en la Figura 1, los pacientes que lograron curación presentaron valores de GH preoperaratorios significativamente más bajos que aquellos no curados. Por otra parte, el punto de corte de $\mathrm{GH}$ basal preoperatoria para predecir curación quirúrgica fue de $15 \mathrm{ng} / \mathrm{ml}$ (sensibilidad 80\%, especificidad 84,9\%, + LR 5,28), lo cual se encuentra graficado en la Figura 2. Al evaluar la variable tamaño tumoral, observamos que el porcentaje de curación fue significativamente menor para macroadenomas versus microadenomas (Figura 3), mientras que no hubo
Figura 1. Esta figura nos muestra la distribución de $\mathrm{GH}$ basal en los pacientes curados y no curados por la cirugía, así como los dos pacientes sin control poscirugía. El grupo curado presentó valores de $\mathrm{GH}$ preoperatoria significativamente menores que el grupo no curado ( $p 0,002)$.

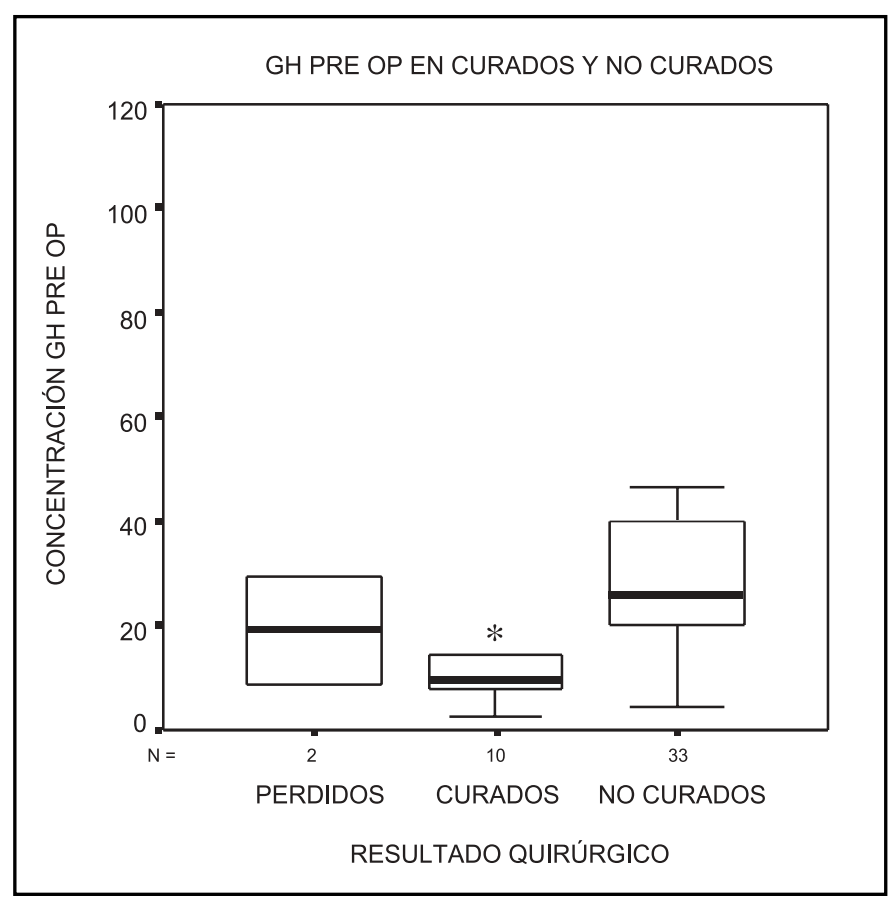




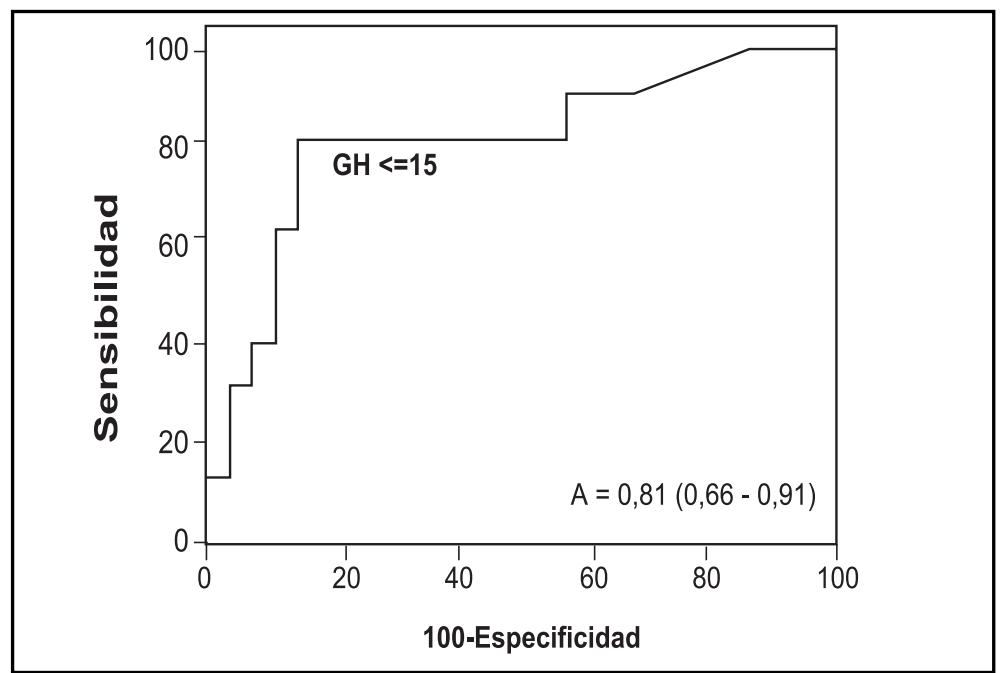

Figura 2. Presentamos la curva ROC para GH basal preoperatoria descrita como predictora de curación quirúrgica y se define como punto de corte GH menor o igual a $15 \mathrm{ng} / \mathrm{ml}$.

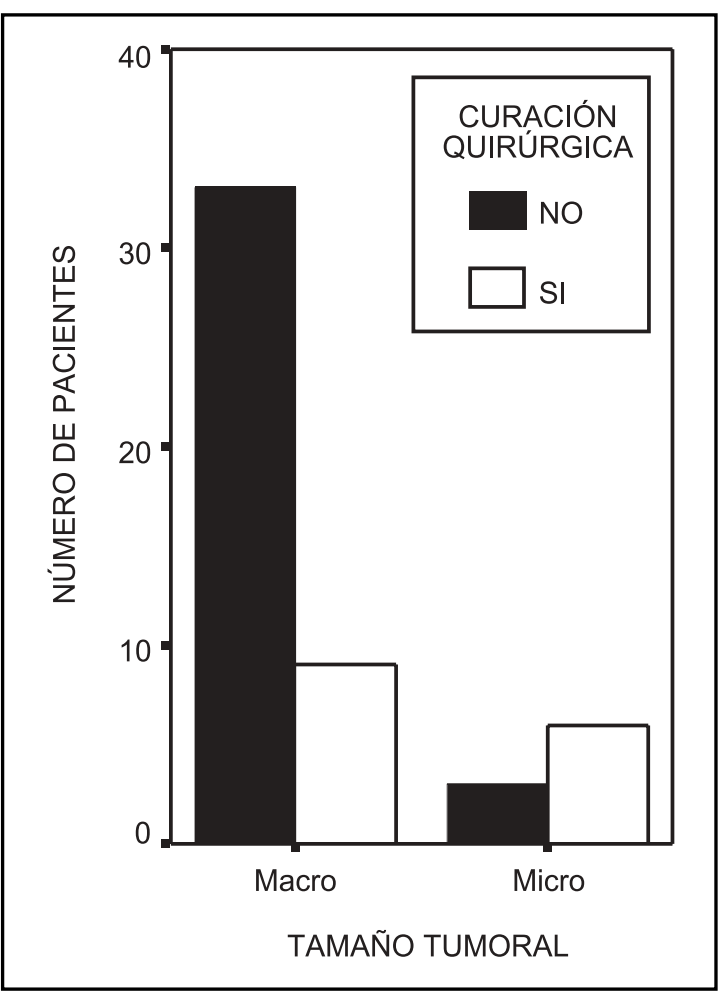

Figura 3. Al comparar la variable curación quirúrgica según tamaño tumoral, observamos que la curación fue más frecuente para los microadenomas $(66,7 \%)$ que para los macroadenomas $(21,4 \%)(\mathrm{p} 0,01)$. diferencias según sexo o edad entre curados y no curados.

En el análisis de regresión logística multivariado que modeló la probabilidad de curación de la cirugía, la única variable que resultó ser significativa fue GH en forma dicotómica (GH $\leq 15 \mathrm{ng} / \mathrm{ml} \mathrm{v} / \mathrm{s}$ $\mathrm{GH}>15 \mathrm{ng} / \mathrm{ml}$ ). La estimación del OR (IC 95\%) fue $22,4(3,63-138,02)$ y el valor $p=0,001$, de lo cual se desprende que los pacientes con GH basal mayor de $15 \mathrm{ng} / \mathrm{ml}$ tenían 22,4 veces más riesgo de no curarse que aquellos con $\mathrm{GH} \leq 15 \mathrm{ng} / \mathrm{ml}$.

De los 15 pacientes que presentaron compromiso visual preoperatorio, $7(46,7 \%)$ recuperaron total o parcialmente el defecto campimétrico, mientras que de los 38 pacientes con estudio oftalmológico normal, uno presentó deterioro posoperatorio persistente. De los 17 pacientes con hipopituitarismo preoperatorio, 3 lograron recuperación de los ejes, mientras que $40 \%$ de los pacientes eupituitarios presentó hipopituitarismo posquirúrgico. El 18,9\% de los pacientes tenía $\mathrm{GH}$ posoperatorio $>20 \mathrm{ng} / \mathrm{ml}$.

Posterior a la cirugía, 26 pacientes fueron enviados a radioterapia convencional por indicación del neurocirujano tratante, con lo cual se logró la curación de 7 pacientes adicionales. La latencia fue de 6 años en promedio (rango: 1,5 a 12 años) con un seguimiento posradioterapia entre 1 y 16 años. 
Las complicaciones quirúrgicas más frecuentes fueron: diabetes insípida transitoria 17,8\%, diabetes insípida persistente 3,5\% y fístula de líquido cefalorraquídeo 3,5\%. Un paciente falleció debido a una meningitis por fístula de líquido cefalorraquídeo posterior a la segunda cirugía. Las complicaciones de la radioterapia más frecuentes fueron: hipopituitarismo 19,2\% y neuritis óptica 2,7\%.

De los 53 pacientes que logramos seguir hasta ahora, dos han fallecido; ambos con acromegalia activa a pesar del tratamiento quirúrgico y de la radioterapia. Una mujer de 66 años falleció de cáncer gástrico a los 6 años de la cirugía pituitaria y un varón de 59 años, falleció de una neumonía intrahospitalaria posterior a un AVE isquémico a los 10 años después de 3 cirugías frustras.

\section{Discusión}

La disponibilidad de mejores técnicas bioquímicas de medición de GH e IGF-I, así como el registro y seguimiento de grandes grupos de pacientes, han permitido un mejor conocimiento de la acromegalia y la creación de nuevos criterios de curación ${ }^{16,17}$. Sin embargo, a pesar de estos avances, el pronóstico de los pacientes sigue dependiendo de la experiencia del neurocirujano tratante, de lo cual se desprende nuestro interés en realizar un análisis crítico de nuestra realidad.

Si comparamos nuestros hallazgos con los publicados en el registro de Drange et $\mathrm{al}^{10}$, nuestra población tiene porcentajes similares de macroadenomas, compromiso neuroftalmológico, así como tipo y tiempo de duración de los síntomas. A diferencia de otras publicaciones, llama la atención en nuestra población la mayor prevalencia en mujeres, el peak más tardío ( $4^{a}$ década) y la mayor participación de neurólogos y ginecólogos en la sospecha diagnóstica. Esto último podńa explicarse por la alta frecuencia de cefalea y la mayor prevalencia en el sexo femenino.

Los objetivos terapéuticos en el manejo de la acromegalia son los de eliminar la morbilidad y recuperar la expectativa de vida, mediante un tratamiento seguro, que permita el control de la masa tumoral y restaure una secreción normal de GH. Desde el punto de vista bioquímico, deben reducirse los valores plasmáticos de IGF-I a los normales para edad y sexo y lograr frenar $\mathrm{GH}$ a menos de $1 \mathrm{ng} / \mathrm{ml}$ durante el TTGO ${ }^{16,17}$.
Independiente del tamaño tumoral, nuestros resultados son claramente insuficientes, con un control de la enfermedad en $66,7 \%$ de los microadenomas y $21,4 \%$ de los macroadenomas. Los reportes de curación de otros centros varían desde $80 \%$ a $100 \%$ para microadenomas y 50 a $65 \%$ para macroadenomas, dependiendo del criterio de curación elegido ${ }^{6,7}$. Al igual que en otros reportes, existen diferencias significativas tanto de tamaño tumoral como de $\mathrm{GH}$ preoperatoria entre curados y no $\operatorname{curados}^{6,7,18}$ y el valor de $\mathrm{GH}$ basal es predictor independiente de curación quirúrgica.

En relación a otras variables, como morbilidad posquirúrgica, grupos extranjeros informan 1 a $2 \%$ de morbilidad mayor (meningitis, fístula de líquido cefalorraquídeo, compromiso de pares craneanos), mientras que nuestro grupo presentó 7,5\% (2 fístula, 1 meningitis y 1 deterioro de la visión) ${ }^{18,19}$. A pesar del tratamiento, en sólo 28,3\% se logró preservar la función pituitaria comparado con $97 \%$ de otros grupos y la recuperación de la visión se logró en $46,7 \%$ comparado con $87 \%$ de otros grupos $8,9,19$. Por lo tanto, independiente del parámetro evaluado, estamos lejos de los resultados de centros de referencia internacionales.

Creemos que estos resultados nos obligan a adoptar una conducta más activa destinada a optimizar un diagnóstico más precoz de la acromegalia, para así detectar la enfermedad en su fase de microadenoma y por ende con valores de GH más bajos, lo cual se correlaciona con mayor probabilidad de curación de la enfermedad ${ }^{6,7,18}$. Esto podría lograrse en base a campañas educativas orientadas a los especialistas a los cuales los pacientes consultan. Un registro nacional de tumores acromegálicos nos permitiría conocer con mayor precisión sus características epidemiológicas, identificar factores pronósticos y realizar un seguimiento prospectivo. Esto podría ayudarnos a definir estrategias de manejo acorde a nuestra realidad.

De nuestro estudio se desprende también que $67,9 \%$ de los pacientes con acromegalia, manejados en un importante centro de referencia, mantienen cifras elevadas y riesgosas de $\mathrm{GH}$ posterior a la cirugía, lo cual implica un aumento de la mortalidad de población laboralmente productiva. Más aún, la efectividad de la radioterapia como tratamiento complementario depende de la $\mathrm{GH}$ posoperatoria, con un quiebre en la posibilidad de 
curación a concentraciones superiores a $20 \mathrm{ng} / \mathrm{ml}^{20}$, lo cual ocurre en $18,9 \%$ de los pacientes operados. Por lo tanto, la realidad local a la cual nos enfrentamos es que cerca de $20 \%$ de los pacientes acromegálicos que manejamos no tiene alternativa de curación real, dado que al fracaso de la cirugía se sumarán lo inalcanzable de la terapia médica con análogos de somatostatina o antagonistas del receptor de GH y la poca efectividad de la radioterapia por lo elevado de la GH posquirúrgica.

Como médicos, debemos recordar la necesidad de evaluar, en forma sistemática, los resultados terapéuticos quirúrgicos y creemos que nuestro trabajo sienta las bases para adoptar un cambio de conducta en el manejo de los tumores pituitarios en Chile, especialmente en los funcionantes y en particular la acromegalia ${ }^{21,22}$. Gittoes et $\mathrm{al}^{6}$ comunicaron que el éxito quirúrgico en

\section{REFERENCIAS}

1. Bengtsson BA, Eden S, Ernest I, Oden A, Sjogren B. Epidemiology and long term survival in acromegaly: a study of 166 cases diagnosed between 1955 and 1984. Acta Med Scand 1988; 223: 32735.

2. Alexander L, Appieton D, Hall R, Ross WM, WILKINSON R. Epidemiology of acromegaly in the Newcastle region. Clin Endocrinol (Oxf) 1980; 12: 71-9.

3. Orme SM, McNaly RJ, Cartwright RA, Belchetz PE. Mortality and cancer incidence in acromegaly: a retrospective cohort study. United Kingdom Acromegaly Study Group. J Clin Endocrinol Metab 1998; 83: 2730-4.

4. Shimatsu A, Yokogoshi Y, Saito S, Shimizu N, Irie M. Long-term survival and cardiovascular complications in patients with acromegaly and pituitary gigantism. J Endocrinol Invest 1998; 21: 55-7.

5. Swearingen B, Barker FG, Katznelson L, Biler B, GrinsPoon S, KLUBANSKI ET AL. Long term mortality after transsphenoidal surgery and adjunctive therapy for acromegaly. J Clin Endocrinol Metab 1998; 83: 3419-26.

6. Gittoes NJL, Sheppard MC, Johnson AP, Stewart PM. Outcome of surgery for acromegaly-the experien- pacientes acromegálicos aumentó de 33 a 66\%, disminuyendo de 8 a 1, el número de neurocirujanos que realizan cirugía de hipófisis. Lissette et al repitieron estos hallazgos ${ }^{23}$. En nuestro grupo, 19 neurocirujanos diferentes realizaron las resecciones tumorales, lo cual podría en parte explicar nuestros magros resultados.

Creemos, por lo tanto, que debemos enfrentar el desafío de crear un centro de derivación de patología pituitaria, donde un número reducido de neurocirujanos logre reunir la casuística de 100 hipófisis de base y 25 anuales para ser catalogados de pituitary surgery ${ }^{24}$. Estamos convencidos que esta medida, junto a la implementación de un registro nacional y el financiamiento por las instituciones de salud del tratamiento médico, nos permitiría optimizar nuestros resultados y mejorar el pronóstico de la acromegalia en Chile.

ce of a dedicated pituitary surgeon. Q J Med 1999; 92: 741-5.

7. Laws ER, Vance ML, Tapar K. Pituitary surgery for the management of acromegaly. Horm Res 2000; 53: 71-5.

8. Pumarino H, Oviedo S, Michelsen H, Campino C. [Active acromegaly and gigantism: some clinical characteristics of 50 patients]. Rev Méd Chile 1991; 119: 897-907.

9. López JM, Méndez J, Mahana D, Vega J, Rodríguez JA, Michaud P ET AL. [Results of transsphenoidal surgery and radiotherapy in the treatment of acromegaly]. Rev Méd Chile 1985; 113: 27-31.

10. Drange M, Fram N, Herman-Bonert V, Melmed S. Pituitary tumor registry: a novel clinical resource. J Clin Endocrinol Metab 2000; 85: 168-74.

11. Gleeson HK, Walker BR, Seckl JR, Padfield PL. Ten years on: Safety of short synacthen tests in assessing adrenocorticotropin deficiency in clinical practice. J Clin Endocrinol Metab 2003; 88: 2106-11.

12. Schiechte JA. Clinical practice: Prolactinoma. N Engl J Med 2003; 349: 2035-41.

13. Beauregard C, Truong U, Hardy J, Serri O. Longterm outcome and mortality after transsphenoidal adenomectomy for acromegaly. Clin Endorinol (Oxf) 2003; 58: 86-91. 
14. Ronchi C, Varca C, Giavoli C, Epaminonda P, BeckPeccoz P, Spada A et al. Long term evaluation of postoperative acromegalic patients in remission with previous and newly proposed criteria. J Clin Endocrinol Metab 2005; 90: 1377-82.

15. Barrande G, Pittino-Lungo M, Coste J, Ponvert D, Bertagna X, Luton J et al. Hormonal and metabolic effects of radiotherapy in acromegaly: long term results in 128 patients followed in a single center. J Clin Endocrinol and Metab 2000; 85: 3779-85.

16. Giustina A, Barkan A, Casanueva FF, Cavagnari F, Frohman L, Ho $\mathrm{K}$ et aL. Criteria for cure of acromegaly: A consensus statement. J Clin Endocrinol Metab 2000; 85: 526-9.

17. Meimed S, Casanueva FF, Cavagnini F, Chanson P, Frohman L, Grossman A ET aL. Guidelines for Acromegaly and Management. J Clin Endocrinol Metabol 2002; 87: 4054-8.

18. KReutzer J, VAnce ML, Lopes MBS, Laws ER. Surgical Management of GH-Secreting Pituitary Adenomas: An Outcome Study Using Modern
Remission Criteria. J Clin Endocrinol Metab 2001; 86: 4072-7.

19. Jane J, ThapAR K, LAws E. Acromegaly: historical perspectives and current therapy. J of neurooncol 2001; 54: 129-37.

20. Barrande G, Pittino-Lungo M, Coste J, Ponvert D, Bertagna X et al. Hormonal and metabolic effects of radiotherapy in acromegaly: long term results in 128 patients followed in a single center. J Clin Endocrinol Metab 2000; 85: 3779-85.

21. Anonymous. First lessons from the «Bristol case». Lancet 1998; 351: 1707.

22. Horton R. How should doctors responds to the GMC's Judgments on Bristol? Lancet 1998; 351: 1900-1.

23. Lissett CA, Peacey SR, Laing I, Tetlow L, Davis JR, Shalet SM. The outcome of surgery for acromegaly: the need for a specialist pituitary surgeon for all types of growth hormone secreting adenoma. Clin Endocrinol (Oxf) 1998; 49: 653-7.

24. Соок DM. AACE Acromegaly Guidelines Task Force. Endocr Pract 2004; 10: 213-25. 